\title{
Differentiated Thyroid Carcinoma: Distant Metastasis as an Unusual Sole Initial Manifestation
}

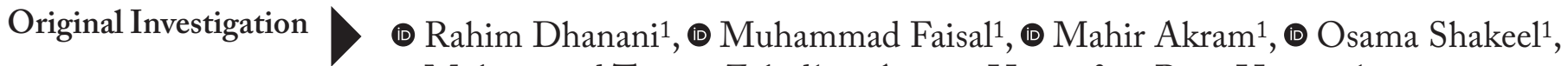 \\ (1) Muhammad Toqeer Zahid¹, (1) Aamna Hassan², (1) Raza Hussain ${ }^{1}$ \\ ${ }^{1}$ Department of Surgical Oncology, Shaukat Khanum Memorial Cancer Hospital \& Research Center, Lahore, Pakistan \\ ${ }^{2}$ Department of Nuclear Medicine, Shaukat Khanum Memorial Cancer Hospital \& Research Center, Lahore, Pakistan
}

\section{ORCID ID of the authors:}

R.D. 0000-0002-5519-862X; M.F. 0000-0001-6564-0410; O.S. $0000-0002-4132-9203$ M.T.Z. 0000-0003-1313-6452; A.H. 0000-0003-0026-0729; R.H. 0000-0002-3552-722X.

Cite this article as: Dhanani R, Faisal $M$, Akram M, Shakeel 0, Zahid MT, Hassan A, Hussain R. Differentiated Thyroid Carcinoma: Distant Metastasis as an Unusual Sole Initial Manifestation. Turk Arch Otorhinolaryngol 2021; 59(3): 188-92

Corresponding Author: Rahim Dhanani; dr.rahimdhanani@gmail.com

Received Date: 12.02 .2021 Accepted Date: 25.05 .2021

Content of this journal is licensed under a Creative Commons Attribution 4.0 International License. Available online at www.turkarchotolaryngol.net

Objective: The objective of this study was to identify the characteristic features of patients with distant metastasis as the only manifestation of well-differentiated thyroid cancers and to analyze the treatment outcomes.

Methods: A retrospective review of all patients with well-differentiated thyroid cancers and distant metastasis as the sole initial presentation was carried out. Data regarding age, gender, tumor histology, site, symptoms, and treatment outcomes were collected.

Results: There were 10 patients who presented with distant metastasis as the only presentation. The mean age was 56.1 years. Eight (80\%) patients had osseous metastasis, one (10\%) had pulmonary and one (10\%) had both. Follicular thyroid carcinoma was more common and seen in six $(60 \%)$ patients. Seven (77.8\%) out of nine patients had demised within five years of initial presentation.

Conclusion: Distant metastases without a neck lump as the initial presentation of welldifferentiated thyroid cancers are extremely rare. No specific guidelines are available to manage such patients due to lack of relevant data in the literature.

Keywords: Differentiated thyroid cancer, neoplasm metastasis, distant metastasis, prognosis, surgery, radioactive iodine, survival

\section{Introduction}

Thyroid cancers account up to $1 \%$ to $5 \%$ of all cancers worldwide (1). The most common endocrine malignancy is thyroid carcinoma which presents as an enlarging lump in the neck (2). The bulk of thyroid malignancies, including papillary and follicular thyroid cancers, have a welldifferentiated form, and usually are clinically indolent with good prognosis (3). The overall survival for 10 years in welldifferentiated thyroid cancers (WDTC) ranges from $85 \%$ to $95 \%$ and drops to $50 \%$ in patients with distant metastasis (4). 
One of the key factors for poor prognosis is distant metastasis; although patients who have WDTC with distant metastasis have comparatively better survival rates than other forms of thyroid cancers (2). Studies have reported 53\% and $58 \%$ five-year survival rates, respectively, in patients with differentiated thyroid cancers with distant metastasis $(5,6)$. This is due to the use of multimodality treatment including surgery, radioactive iodine, radiotherapy and surgical removal of metastatic deposit depending on the type and site of the metastasis $(5,7)$. Most of the times, thyroid carcinoma metastases remain asymptomatic and are only discovered on surveillance or whole-body metastatic work up (2). However, symptomatic distant metastasis can rarely present as an unusual and sole initial presentation of WDTC without any swelling in the neck $(2,8)$.

Because of the rarity of such cases, there is limited data available on this subject in literature and the course of disease as well as the treatment outcomes in these patients are not well documented. Therefore, we decided to describe the treatment outcomes of such patients treated at our center; hence, the objective of our study was to report and identify the characteristic features of the patients who presented with distant metastasis as the only manifestation of WDTC and analyze the treatment outcomes.

\section{Methods}

A retrospective review of all patients treated for WDTC at a dedicated cancer center between 1995 to 2015 was carried out. Approval was obtained from the Institutional Review Board (IRB) of the Shaukat Khanum Memorial Cancer Hospital \& Research Centre, Lahore, Pakistan with IRB number EX-15-07-20-01 and written informed consent was taken from all the patients. All patients with cytologically or histologically proven diagnosis of WDTC and presenting with distant metastasis as the sole initial presentation without any complaint of neck swelling or goiter were included. Patients with simultaneous distant metastasis and thyroid or neck swelling, or those with missing data were excluded. Cervical lymph node metastasis was not considered as distant metastasis. Data regarding age, gender, tumor histology, site, symptoms and treatment outcomes were collected. The data were analyzed using SPSS software v25.0.

\section{Results}

The total number of patients with WDTC managed between 1995 to 2015 were 886 (papillary thyroid cancer = 689 , follicular thyroid cancer $=114$ and medullary thyroid cancer $=83)$. Out of these, $76(8.6 \%)$ patients presented with metastasis at presentation. Ten patients were identified to fulfill the inclusion criteria of presenting with distant metastasis as the only presentation without any prior history of thyroid or neck lump (Figures 1 and 2). The mean age at presentation was $56.1 \pm 10.6$ years (range: $36-78$ years). There were seven $(70 \%)$ female and three $(30 \%)$ male patients.

All patients were symptomatic at the time of presentation, examinations, including fine needle aspiration cytology (FNAC) or incisional biopsies of the metastatic lesion were performed, led to the final diagnosis of differentiated thyroid cancer with distant metastasis. The most common

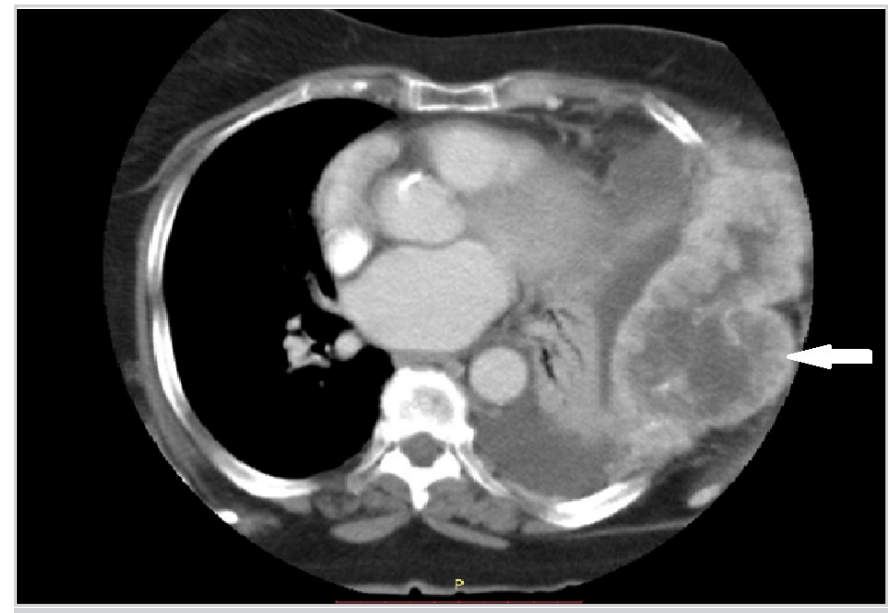

Figure 1. Papillary carcinoma of thyroid with follicular variant. CT of thorax showing the destructive lesion in left lower rib cage with large soft tissue component measuring $13.3 \times 6.5 \mathrm{~cm}$ (arrow)

CT: Computed tomography

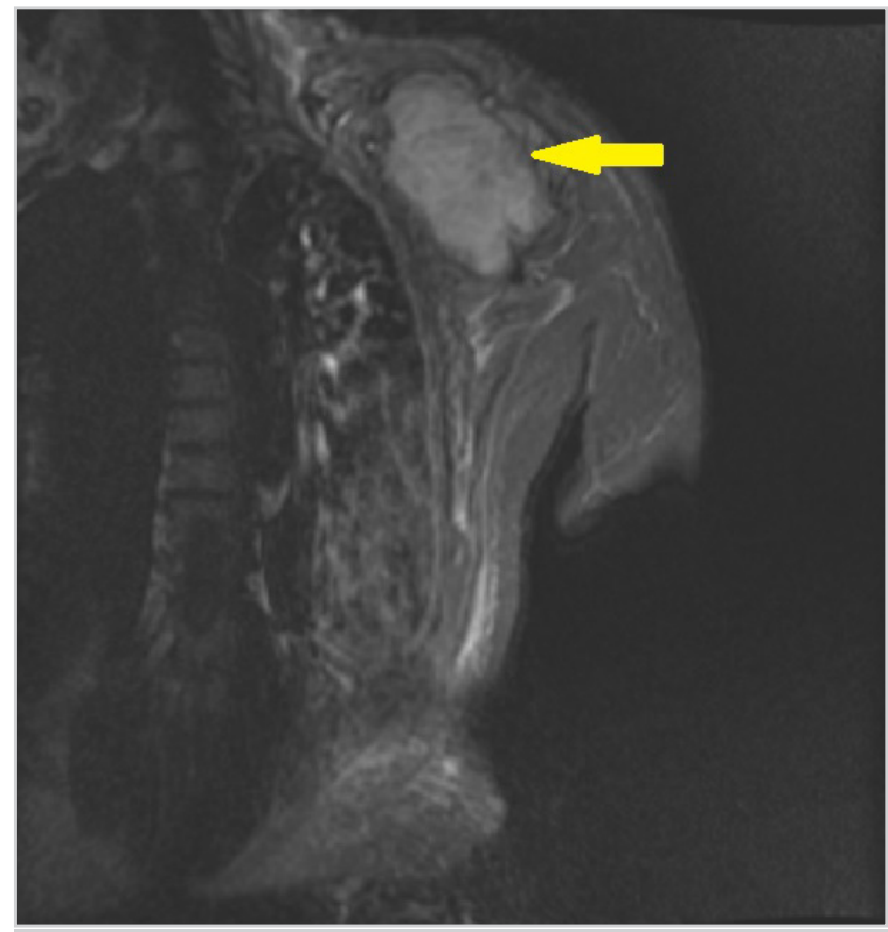

Figure 2. Follicular neoplasm of thyroid. MRI of upper limb shows large lobulated soft tissue mass centered on left scapula associated with its erosion and destruction (arrow)

MRI: Magnetic resonance imaging 
initial presentation was bone pain or fracture, seen in five (50\%) patients, followed by mass or swelling seen in three (30\%) patients. Two (20\%) patients presented with more than one symptom, one of them presented with hoarseness and difficulty in breathing whereas the other presented with difficulty in breathing along with pain in sternal bone. Eight (80\%) patients had osseous metastasis, one (10\%) had pulmonary and one (10\%) had both pulmonary and osseous metastasis. In nine patients with osseous involvement, the appendicular skeleton was more commonly involved and seen in six (66.7\%) patients whereas osseous metastasis was in the axial skeleton in three (33.3\%) patients.

Regarding histology, follicular thyroid carcinoma was seen in six $(60 \%)$ patients of whom five had extracapsular extension and angioinvasion. The remaining four (40\%) patients were diagnosed with papillary thyroid carcinoma, two of which were follicular variant. There were six $(60 \%)$ patients who underwent surgery. Five (50\%) patients underwent total thyroidectomy. Of these, one patient also underwent lateral neck dissection. There was one patient who underwent wide excision of sternal mass. Adjuvant radioactive iodine (RAI) was given to six (60\%) patients $(200 \mathrm{mci}$ in three patients and $150 \mathrm{mci}$ in the other three patients); out of these, four (66.7\%) patients received multiple sessions of RAI (200 mci in two patients and $150 \mathrm{mci}$ in two patients). Post therapy thyroglobulin and antithyroglobulin levels were raised in four patients. Eight (80\%) patients received radiation therapy and majority of them $(n=5,62.5 \%)$ were given radiation with a palliative intent.

All patients were followed up regularly, except for one patient who was lost to follow-up. Mean follow-up period was 41.7 months (minimum one month and maximum 156 months). Seven (77.8\%) out of nine patients died within the five years after initial presentation. Except for one case, all deaths were related to the disease process. The characteristics of all patients included in the study are provided in Table 1.

\section{Discussion}

WDTCs limited to thyroid gland have an excellent survival outcome, with a 10-year disease-specific survival outcome reported up to $90 \%$ (9). Even in the presence of distant metastasis, it has a better survival outcome compared to the other malignancies with distant metastasis $(5,6)$. WDTCs are more often limited to thyroid gland only, and distant metastasis is rare, with rates reported from $1 \%$ to $15 \%$ in the literature (10-12). The prevalence of distant metastasis in WDTC was reported up to $2.2 \%$ in a comprehensive Surveillance, Epidemiology, and End Results (SEER) database study (13). The most common sites of distant metastasis are the bones and the lungs, although involvement of other organs is also reported in the data, at a rate less than $5 \%(4,8,12)$. In our series, eight $(80 \%)$ patients had osseous metastasis, one (10\%) had pulmonary and one (10\%)

Table 1. Characteristics of patients with distant metastasis as the sole initial presentation of differentiated thyroid cancers

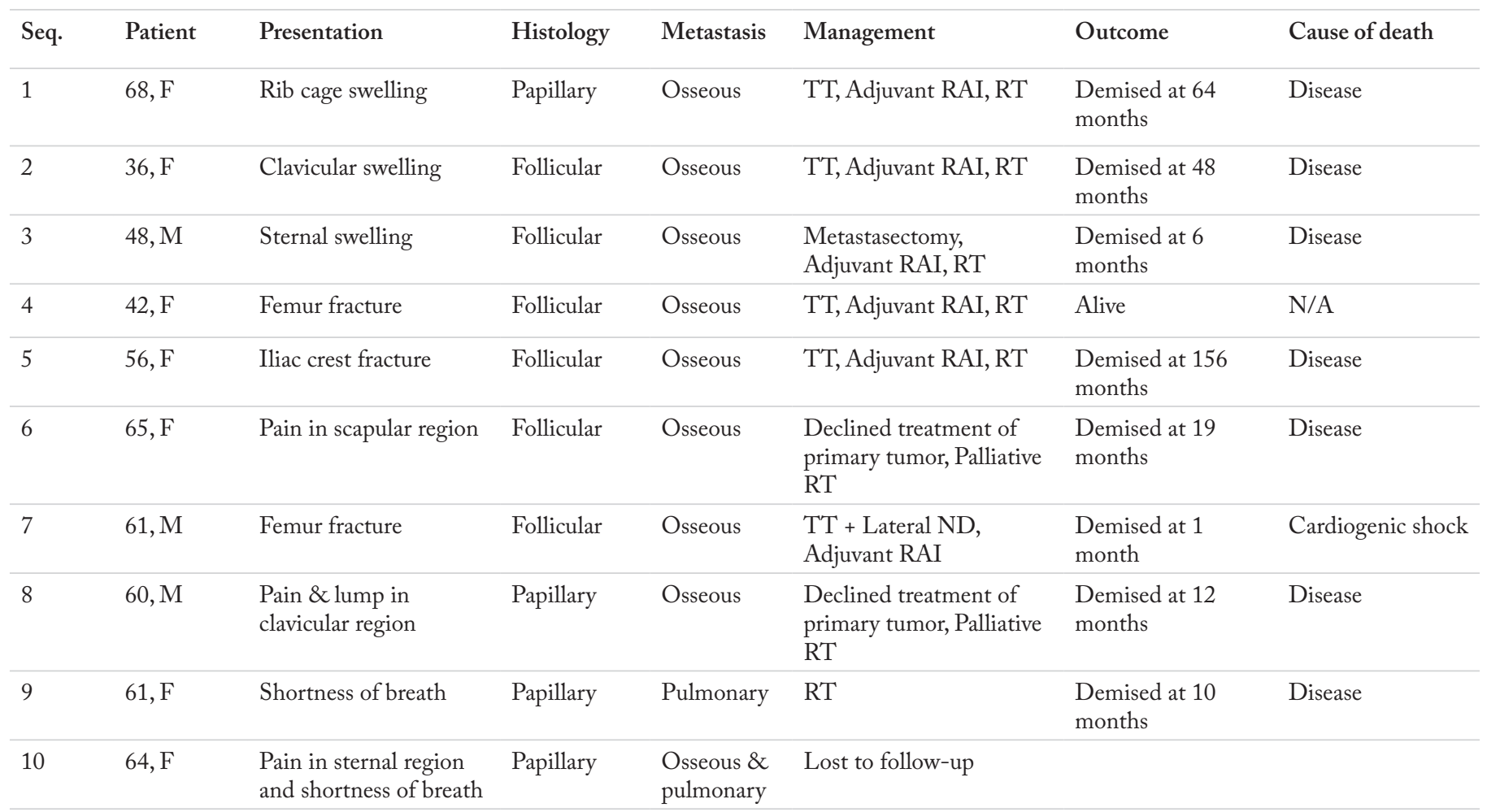

F: Female, M: Male, TT: Total thyroidectomy, ND: Neck dissection, RAI: Radioactive iodine, RT: Radiation therapy, Seq: Sequence 
had combination of pulmonary and osseous metastasis, these findings are similar to the data reported in the literature (2, 8).

Distant metastasis at the time of initial presentation has a significant prognostic implication as the 10-year survival rate falls significantly to $50 \%$. Factors including age, gender and distant metastasis are associated with survival outcomes in patients with $\operatorname{WDTC}(12,14,15)$. Metastasis to organs other than the bones and the lungs is not well understood and usually missed in clinical settings because of the rarity of such cases. Shaha et al. (11) reported a case series of 44 patients, over a period of more than half a century, with distant metastases as the only initial presenting symptom. Since then, the literature regarding this topic has remained scarce.

Shaha et al. (11) reported that acceptable long-term survival outcomes were achieved after adequate treatment of primary tumor and aggressive RAI for metastatic disease. Similar management approach is reported in our series and in the literature $(2,8)$.

See et al. (2) found that follicular thyroid carcinoma, which has hematogenous spread, was most frequently associated with patients with distant metastasis followed by papillary thyroid carcinoma which has lymphatic spread. Also, they found that papillary thyroid carcinoma was more frequently associated with multiple foci of distant metastasis as compared to follicular thyroid carcinoma. In our series we had similar findings that were found comparable with data available in the literature $(11,16)$.

Mazzaferri (17) took 40 years of age as cut-off and found that patients younger than 40 had a significantly lower risk of developing distant metastasis compared to patients older than 40. Similar findings were noted in our study. The mean age of our patients at the time of their presentation was 56.1 years and only one patient was younger than 40 . Comparable results are seen in studies reported in the literature $(2,8,18)$.

Patients with swelling in the neck, an easily noticeable and palpable region of the body, would normally seek early medical advice. In our series, patients did not present to head and neck surgeons due to the atypical initial presentation and usually sought medical advice from other specialty departments for complaints such as bone pain, bone fracture and difficulty in breathing, leading to delays and providing the disease the opportunity to metastasize further and thereby adversely affecting the survival outcomes. Studies in the literature also reported similar trends of presentation, with patients being referred to head and neck surgeons by other specialty departments $(2,8)$.

The American Thyroid Association (ATA) guidelines are widely accepted and used in the management of nonmetastatic WDTC (2). There is, however, lack of consensus as to which management protocols should be opted for patients with metastatic thyroid cancer because of the paucity of data on thyroid cancers with distant metastasis. The management plan for all the patients included in our study was discussed and decided by the Multidisciplinary Tumor Board and we advocate that a similar approach is adopted for all patients with rare and atypical presentation.

Our study has several limitations, namely the small sample size, single institution experience and retrospective study design. We were not able to identify the prognostic and risk factors because of our smaller sample size. Nonetheless, the findings of our study on the characteristic features of a rare entity can help further investigations on the risk and prognostic factors in patients diagnosed with WDTC, a condition which generally is regarded as a relatively favorable condition but may have a poor clinical outcome.

\section{Conclusion}

We conclude that distant metastases without a neck mass as the sole initial presentation of WDTC is extremely rare. While follicular thyroid carcinoma is the most common etiology, osseous metastases are seen more commonly. Its prognosis is low. Due to the paucity of data, there are still no guidelines available for the treatment of such patients. We recommend further studies in order to better understand the natural course of the disease and subsequently to formulate the guidelines that will help clinicians devise a correct management plan when dealing patients with thyroid cancers with distant metastases.

Ethics Committee Approval: Approval from Institutional Review Board (IRB) of Shaukat Khanum Memorial Cancer Hospital and Research Centre was taken. IRB number: EX15-07-20-01.

Informed Consent: Written informed consent was taken from all the patients included in the study.

Peer-review: Externally peer-reviewed.

Conflict of Interest: The authors declare no conflict of interest.

Financial Disclosure: The authors declare that this study received no financial support.

\section{Authorship Contributions}

Surgical and Medical Practices: A.H., R.H., Concept: R.D., M.F., A.H., R.H., Design: R.D., M.F., O.S., M.T.Z., Data Collection and/or Processing: R.D., M.A., O.S., M.T.Z., Analysis and/or Interpretation: R.D., M.F., O.S., M.T.Z., A.H., R.H., Literature Search: R.D., M.F., M.A., O.S., M.T.Z., Writing: R.D., M.F., M.A., A.H., R.H. 


\section{Main Points}

- The incidence of thyroid cancers is on a rising trend, mainly as a result of the advancement in diagnostic modalities.

- Distant metastasis in well-differentiated thyroid cancers is rarely seen and its presentation without any neck mass is even rarer.

- Here, we report an interesting investigation on the characteristic features of the patients who had presented with distant metastasis as the only manifestation of well-differentiated thyroid cancers, and their treatment outcomes.

- These patients have low prognosis. Due to paucity of data, there are still no guidelines available to treat such patients.

\section{References}

1. Kilfoy BA, Zheng T, Holford TR, Han X, Ward MH, Sjodin A, et al. International patterns and trends in thyroid cancer incidence, 1973-2002. Cancer Causes Control 2009; 20: 525-31. [Crossref]

2. See A, Iyer NG, Tan NC, Teo C, Ng J, Soo KC, et al. Distant metastasis as the sole initial manifestation of well-differentiated thyroid carcinoma. Eur Arch Otorhinolaryngol 2017; 274: 287782. [Crossref]

3. Hundahl SA, Fleming ID, Fremgen AM, Menck HR. A National Cancer Data Base report on 53,856 cases of thyroid carcinoma treated in the U.S., 1985-1995. Cancer 1998; 83: 2638-48. [Crossref]

4. Madani A, Jozaghi Y, Tabah R, How J, Mitmaker E. Rare metastases of well-differentiated thyroid cancers: a systematic review. Ann Surg Oncol 2015; 22: 460-6. [Crossref]

5. Schlumberger M, Tubiana M, De Vathaire F, Hill C, Gardet P, Travagli JP, et al. Long-term results of treatment of 283 patients with lung and bone metastases from differentiated thyroid carcinoma. J Clin Endocrinol Metab 1986; 63: 960-7. [Crossref]

6. Mihailovic JM, Stefanovic LJ, Malesevic MD, Erak MD, Tesanovic DD. Metastatic differentiated thyroid carcinoma: clinical management and outcome of disease in patients with initial and late distant metastases. Nucl Med Commun 2009; 30: 558-64. [Crossref]

7. Huang IC, Chou FF, Liu RT, Tung SC, Chen JF, Kuo MC, et al. Long-term outcomes of distant metastasis from differentiated thyroid carcinoma. Clin Endocrinol (Oxf) 2012; 76: 439-47. [Crossref]
8. Iftikhar H, Ikram M, Muhammad AY, Nathani KR. Unusual presentation of differentiated thyroid cancer metastasis. Int Arch Otorhinolaryngol 2018; 22: 167-70. [Crossref]

9. Lang BH, Wong KP, Cheung CY, Wan KY, Lo CY. Evaluating the prognostic factors associated with cancer-specific survival of differentiated thyroid carcinoma presenting with distant metastasis. Ann Surg Oncol 2013; 20: 1329-35. [Crossref]

10. Mihailovic J, Stefanovic L, Malesevic M. Differentiated thyroid carcinoma with distant metastases: probability of survival and its predicting factors. Cancer Biother Radiopharm 2007; 22: 250-5. [Crossref]

11. Shaha AR, Shah JP, Loree TR. Differentiated thyroid cancer presenting initially with distant metastasis. Am J Surg 1997; 174: 474-6. [Crossref]

12. Song HJ, Xue YL, Xu YH, Qiu ZL, Luo QY. Rare metastases of differentiated thyroid carcinoma: pictorial review. Endocr Relat Cancer 2011; 18: R165-74. [Crossref]

13. Goffredo P, Sosa JA, Roman SA. Differentiated thyroid cancer presenting with distant metastases: a population analysis over two decades. World J Surg 2013; 37: 1599-605. [Crossref]

14. Hassan A, Khalid M, Riaz S, Nawaz MK, Bashir H. Follicular thyroid carcinoma: disease response evaluation using American Thyroid Association risk assessment guidelines. Eur Thyroid J 2015; 4: 260-5. [Crossref]

15. Hassan A, Razi M, Riaz S, Khalid M, Nawaz MK, Syed AA, et al. Survival analysis of papillary thyroid carcinoma in relation to stage and recurrence risk: a 20-year experience in Pakistan. Clin Nucl Med 2016; 41: 606-13. [Crossref]

16. Ruegemer RJJ, Hay ID, Bergstralh EJ, Ryan JJ, Offord KP, Gorman CA. Distant metastases in differentiated thyroid carcinoma: a multivariate analysis of prognostic variables. J Clin Endocrinol Metab 1988; 67: 501-8. [Crossref]

17. Mazzaferri EL. An overview of the management of thyroid cancer. Mazzaferri EL, Harmer C, Mallick UK, Kendall-Taylor P, editors. Practical management of thyroid cancer. London: Springer; 2006. p.1-28. [Crossref]

18. Lee J, Soh EY. Differentiated thyroid carcinoma presenting with distant metastasis at initial diagnosis: clinical outcomes and prognostic factors. Ann Surg 2010; 251: 114-9. [Crossref] 